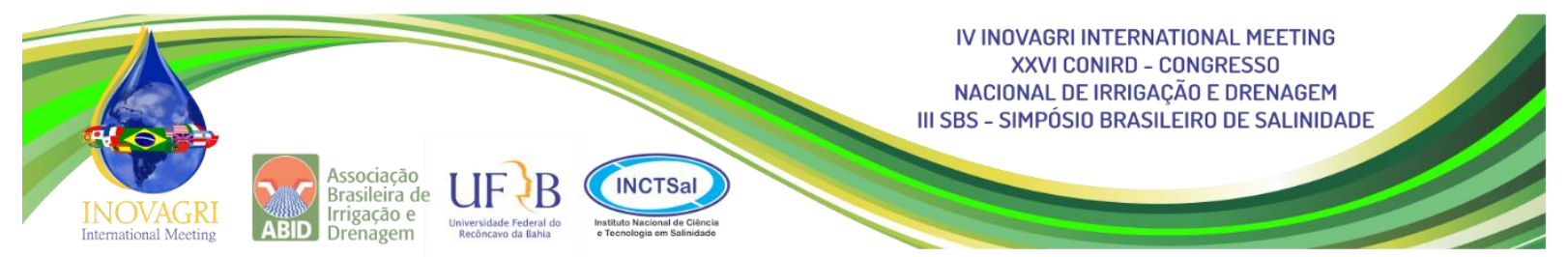

\title{
PACLOBUTRAZOL APLICADO VIA SISTEMA DE IRRIGAÇÃO NA INDUÇÃO FLORAL DA MANGUEIRA 'KEITT'
}

\author{
W. L. Simões ${ }^{1}$, M. A. de $\operatorname{Souza}^{2}$, M. A. do C. $\mathrm{Mouco}^{3}$, M. A. C. de Lima ${ }^{4}$ M. Calgaro ${ }^{5}$
}

RESUMO: O paclobutrazol (PBZ) vem sendo utilizado para auxiliar na indução floral da mangueira nos perímetros irrigados. Entretanto, sua aplicação tem sido feita de forma manual, resultando em baixa uniformidade na distribuição e perdas com mão-de-obra. O objetivo deste trabalho foi ajustar a aplicação do PBZ via sistema de irrigação para o cultivo da mangueira 'Keitt'. O experimento foi conduzido na fazenda Special Fruit, localizada em Petrolina-PE. O delineamento experimental foi em blocos casualizados, com seis tratamentos, cinco doses de $\operatorname{PBZ}\left(0,7 ; 0,9 ; 1,1 ; 1,3 ; 1,5\right.$ g.i.a.m ${ }^{-1}$ linear de copa) aplicadas via sistema de irrigação, e uma dose aplicada de forma convencional (1,3 g.i.a.m ${ }^{-1}$ linear de copa), com quatro repetições. A aplicação via sistema de irrigação na dose igual e superior a aplicada da forma convencional proporcionou produtividade inferior. A produtividade, número de frutos e a densidade dos frutos tiveram uma redução com o aumento da concentração do produto, o que pode estar relacionado com a condutância estomática e a fotossíntese que também apresentaram diferença entre os tratamentos. A dose de 0,7 g i.a.m ${ }^{-1}$ (46\% da dose aplicada de forma convencional) proporcionou um aumento de $32 \%$ na produtividade da mangueira 'Keitt'.

PALAVRAS-CHAVES: PBZ, fisiologia, pós-colheita

\section{PACLOBUTRAZOL BY IRRIGATION ON MANGO 'KEITT' FLORAL INDUCTION}

\begin{abstract}
Paclobutrazol (PBZ) has been used to mango floral induction in the irrigated perimeters. However, its application has been done manually, resulting in a low uniformity of application and losses with labor. Aim of this work was to adjust the application of PBZ via irrigation system for the cultivation of Keitt hose. The experiment was conducted at the Special Fruit farm, located in Petrolina-PE. The experimental design was a randomized complete block
\end{abstract}

\footnotetext{
${ }^{1}$ Engenheiro Agrônomo, pesquisador da Embrapa Semiárido, e-mail: welson.simoes@embrapa.br;

${ }^{2}$ Biólogo; Doutorando em Fisiologia na UFLA; e-mail: moisesalves-1989@ hotmail.com

${ }^{3}$ Engenheira Agrônoma, pesquisadora da Embrapa Semiárido, e-mail:maria.mouco@embrapa.br

${ }^{4}$ Engenheira Agrônoma, pesquisadora da Embrapa Semiárido, e-mail:auxiliadora.lima@embrapa.br

${ }^{5}$ Engenheiro Agrônomo, pesquisador da Embrapa Semiárido, e-mail: marcelo.calgaro@embrapa.br
} 
design with five doses of PBZ, applied through an irrigation system $(0.7,0.9,1.1,1.3,1.5 \mathrm{~g}$ of i.a. per linear meter of canopy), and a manually applied dose to the haul (1.3 $\mathrm{g}$ of i.a. per linear meter of canopy) with four replicates. The application via the irrigation system at the same and superior dosage applied in the conventional manner provided lower productivity. Productivity, number of fruits and denso had a reduction with the increase of the product concentration, which may be related to stomatal conductance and photosynthesis, which also presented a difference between the treatments. The dosage of 0.7 g.i.a.m.-1 (46\% at the dosage applied in the conventional manner) provided a $32 \%$ increase in productivity of mango 'Keitt'.

KEYWORDS: PBZ, physiological, postharvest

\section{INTRODUÇÃO}

O potencial existente para a exploração da fruticultura irrigada faz desta atividade um ótimo negócio para o desenvolvimento da agricultura na região nordeste e, consequentemente, para a economia do país (OLIVEIRA; FARIAS FILHO, 2013). Entre as culturas exploradas, a mangueira (Mangifera indica L) tem sido responsável por um dos melhores desempenhos, sendo em 2014 a segunda fruta mais exportada em volume pelo Brasil, com ampliação do embarque de 9,04\%, em relação ao ano anterior, enviando 133,03 mil toneladas e sendo a primeira em receita gerada (US\$ 163,73 milhões) (ANUARIO BRASILERO DE FRUTICULTURA, 2015).

A manipulação da época de produção da mangueira por meio de práticas como manejo da irrigação, podas e uso de reguladores vegetais, associadas às condições climáticas adequadas, tem possibilitado aos produtores de manga dessa região o escalonamento da produção, em função da demanda do mercado (RAMIREZ; DAVENPORT, 2010).

A indução floral da cultura tem sido realizada com o auxílio do regulador vegetal paclobutrazol (PBZ), que regula o crescimento vegetativo, função do efeito na inibição da biossíntese das giberelinas. Na prática, tem-se observado que a dose e a forma de aplicação do PBZ influenciam significativamente a resposta das plantas (MOUCO et al., 2010).

O PBZ tem sido aplicado de forma convencional no cultivo da mangueira, por meio da diluição do produto comercial em água, que é despejado ao solo, junto ao colo ou na projeção da copa. No intuito de reduzir os custos com mão-de-obra, a aplicação via sistema de irrigação tem sido testado por alguns produtores, porém, sem nenhuma validação técnica na região do Submédio do São Francisco. 
Neste contexto, objetivou-se com o presente trabalho, ajustar a dose ideal do PBZ a ser aplicada via sistema de irrigação para manejo da produção e qualidade de frutos na mangueira cultivar Keitt, na região do Submédio do Vale do São Francisco.

\section{MATERIAL E MÉTODOS}

O experimento foi conduzido em um pomar de mangueiras da cultivar Keitt, na Fazenda Special Fruit, no município de Petrolina-PE, localizada a $9^{\circ} 8^{\prime} 8,9^{\prime \prime} \mathrm{S}$ de latitude, $40^{\circ} 18^{\prime} 33,6^{\prime \prime} \mathrm{O}$ de longitude e $373 \mathrm{~m}$ de altitude. O clima da região é classificado como semiárido, do tipo BSwh’ segundo a classificação climática de Köppen. As chuvas se concentram nos meses de novembro a abril, com precipitação média anual em torno de $500 \mathrm{~mm}$, irregularmente distribuída. A umidade relativa média anual é de $66 \%$ e a temperatura média do ar anual é de $26,5^{\circ} \mathrm{C}$.

Foram utilizadas plantas de mangueira da cultivar Keitt, com seis anos de idade, em um espaçamento de $6 \times 2 \mathrm{~m}$, com um diâmetro médio da copa de $2 \mathrm{~m}$. A irrigação da área foi feita por meio de duas linhas de gotejo por fileira de planta, em um espaçamento de 0,50 $\mathrm{m}$ entre gotejadores. Os tratos culturais como capina, adubação e pulverizações com defensivos, foram os normalmente utilizados na propriedade e preconizadas por Albuquerque et al., (2002). A fonte de PBZ foi o Cultar, produto comercial da Syngenta, com $25 \%$ de ingrediente ativo (i.a.).

O delineamento experimental utilizado foi em blocos casualizados, com seis tratamentos, cinco doses de PBZ aplicados via sistema de irrigação (T1 - 0,7; T2 - 0,9; T3 - 1,1; T4 - 1,3; e T5 - 1,5 g.i.a.m ${ }^{-1}$ linear de copa), e um tratamento adicional, com a aplicação de uma dose na forma convencional (T0-1,3 g.i.a.m-1 linear de copa), com quatro repetições. As parcelas foram constituídas por quatro plantas sendo consideradas as duas plantas centrais como úteis.

As plantas foram podadas e, depois da emissão de dois fluxos vegetativos, a aplicação do PBZ foi feita na forma e doses previstas nos tratamentos. Foram tomados os dados de número de frutos por planta, produtividade total e os referentes a qualidade pós-colheita: diâmetro do fruto, com o paquímetro digital; firmeza da polpa $(\mathrm{N})$, com o penetrômetro; sólidos solúveis, por refratometria; acidez titulável, titulando-se $1 \mathrm{~g}$ de suco diluído em (50) mL de água destilada, com $\mathrm{NaOH}(0,1)$, e o $\mathrm{pH}$, determinado no extrato aquoso com auxílio de um potenciômetro de mesa.

As avaliações fisiológicas foliares foram realizadas no estádio de frutificação da mangueira, em que as trocas gasosas foram avaliadas a partir do Analisador Portátil de Gás 
Infravermelho (IRGA), modelo Li-6400, utilizando luz artificial fixada em $2500 \mu \mathrm{mol} \mathrm{m}^{-2} \mathrm{~s}^{-1}$. As variáveis analisadas foram: taxa de fotossíntese $(A)$, condutância estomática (gs), transpiração (E) e temperatura foliar (Tf). As amostragens foram realizadas em folhas expostas ao sol, no horário entre 9:00 e 11:00 h.

Os dados foram submetidos à análise de variância, sendo as médias submetidas ao teste de Tukey (5\%) para comparar as duas formas de aplicação do PBZ e regressão para as doses aplicadas via sistema de irrigação, quando significativamente diferentes. As análises foram feitas com o auxílio do programa SISVAR® 3.01.

\section{RESULTADOS E DISCUSSÃO}

Observou-se na análise de variância que houve diferença significativa para as variáveis número de frutos por planta e produtividade, entre as doses de paclobutrazol (PBZ) aplicadas via sistemas de irrigação. Para os dados referentes a pós-colheita, foram observadas diferenças entre os tratamentos para a variável densidade dos frutos.

Avaliando-se a influência das doses de PBZ aplicadas via sistema de irrigação sobre o número médio de frutos e a produtividade total, observou-se que houve um decréscimo com o aumento das doses de PBZ aplicada. A dose 0,7 g.i.a. PBZ $\mathrm{m}^{-1}$ linear de copa proporcionou maior número de frutos por planta (140) e produtividades $\left(35,8 \mathrm{t} \mathrm{Ha}^{-1}\right)$ entre os tratamentos, conforme pode ser observado nas Figuras 1A e 1B, respectivamente.

Chatzivagiannis et al. (2014) observaram um decréscimo significativo no número de frutos quando doses acima de $0,8 \mathrm{~g}$ i.a $\mathrm{PBZ} \mathrm{m}^{-1}$ linear de copa foram aplicadas de forma convencional em mangueiras 'Boubon', 'Palmer' e 'Rosa'. O mesmo comportamento foi observado por Cardoso et al. (2007), avaliando o florescimento e frutificação da cultivar Rosa em diferentes doses de PBZ aplicadas de forma convencional. Albuquerque et al (2002), nas condições do Vale do São Francisco, sugerem que para a cultivar Tommy Atkins as doses de PBZ, aplicadas de forma convencional, variem entre 0,5 e $1,0 \mathrm{~g}^{\mathrm{i} . \mathrm{a} \mathrm{m}} \mathrm{m}^{-1}$ linear de copa e que essas devem ser adequadas, principalmente, às condições de solo, clima (época de indução). As doses recomendadas de PBZ são citadas por Ferrari e Sergent (1996) na faixa de 2,5 a 3,75 g i.a /planta, para mangueiras entre 3 e 4 anos e de 5,0 a 10,0 g i.a para plantas com mais de cinco anos. Os resultados encontrados demonstram que é possível reduzir a dose de PBZ aplicada em até $46 \%$, quando aplicado via sistema de irrigação, obtendo-se ainda um aumento de $32 \%$ na produtividade da mangueira 'Keitt'. 

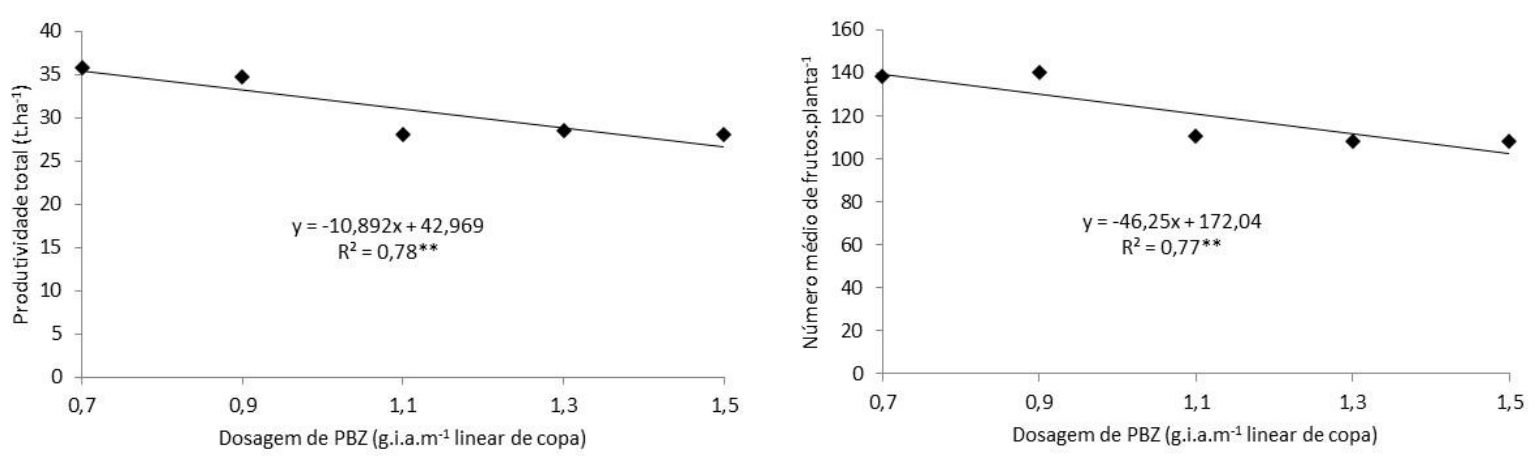

Figura 1. Produtividade total (A) e número médio de frutos (B) da mangueira 'Keitt' em função de doses de paclobutrazol aplicadas via sistema de irrigação, na região do Submédio do São Francisco.2016. Petrolina/PE.

Semelhante ao observado para número de frutos por planta e produtividade, na avaliação pós colheita observou-se que a densidade dos frutos também decresceu com o aumento da dose de PBZ (Figura 2), sendo encontrada a maior densidade de 2,47 para dose de 0,7 g.i.a.m ${ }^{-1}$ linear de copa.

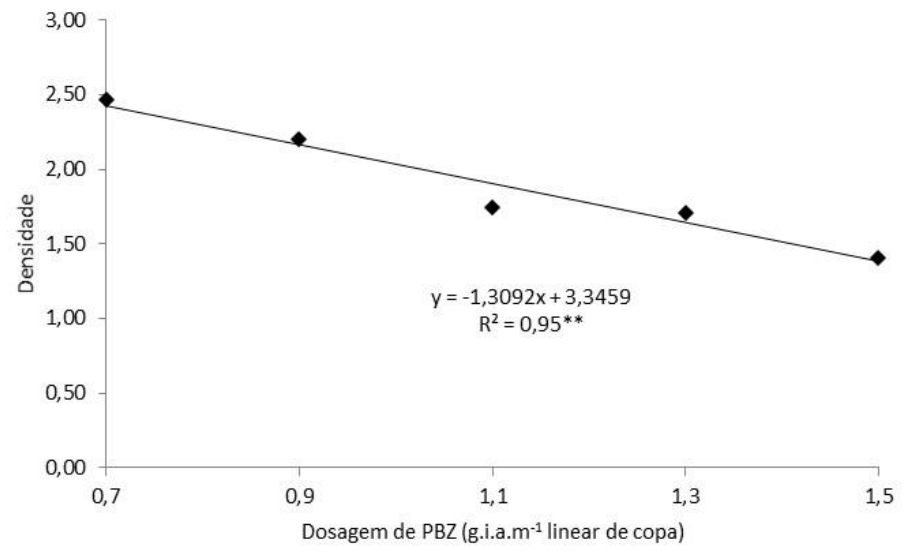

Figura 2. Densidade dos frutos da mangueira cultivar Keitt, em função de doses de paclobutrazol aplicadas via sistema de irrigação, na região do Submédio do São Francisco. Petrolina/PE.

Não houve diferença para firmeza da polpa da manga, sólidos solúveis e acidez titulável, sendo os valores médios encontrados de $11,49 \mathrm{~N}, 7,41^{\circ}$ Brix e 1,63\%, respectivamente.

Reis et al. (2011), avaliando o efeito de lâminas de irrigação e doses de PBZ na póscolheita da mangueira cv. Haden, encontraram os valores médios do teor dos sólidos solúveis de $5,85^{\circ}$ Brix e da acidez titulável, 0,14\%, menores dos encontrados neste trabalho e devido provavelmente a que se trata de uma outra cultivar e pelo ponto de maturação do fruto na colheita. 
Comparando-se a aplicação do PBZ via sistema de irrigação e via convencional, na mesma dose, não foi observada diferença significativa para número de frutos por planta, produtividade $\left(\mathrm{t} \mathrm{ha}^{-1}\right)$ e características de qualidade dos frutos na pós-colheita (Tabela1).

Tabela 1. Número de frutos por planta, produtividade $\left(\mathrm{t} \mathrm{ha}^{-1}\right)$, densidade, firmeza $(\mathrm{N})$, sólidos solúveis (SS) e acidez titulável (AT) dos frutos, em mangueira 'Keitt', em função da forma de aplicação do paclobutrazol, no Submédio do São Francisco. Petrolina/PE.

\begin{tabular}{ccccccc}
\hline \multirow{2}{*}{ Formas de aplicação } & $\begin{array}{c}\text { Produtividade } \\
\mathrm{t} \mathrm{ha}^{-1}\end{array}$ & $\begin{array}{c}\text { Frutos por } \\
\text { planta }\end{array}$ & Densidade & $\mathrm{N}$ & Brix & $\begin{array}{c}\text { AT } \\
\%\end{array}$ \\
\hline Convencinal & 31,30 & 117,67 & 1,70 & 11,69 & 7,00 & 1,69 \\
Via sist. irrigação & 28,50 & 108,50 & 1,71 & 11,39 & 7,13 & 1,52 \\
\hline
\end{tabular}

Verificou-se na análise de variância que houve diferença significativa para as características fisiológicas: transpiração, condutância estomática e fotossíntese (Figuras 2A, 2B e $2 \mathrm{C}$, respectivamente) em função das doses de PBZ aplicadas via sistema de irrigação, não havendo para temperatura foliar (valor médio $30,6^{\circ} \mathrm{C}$ ). De modo geral, a dose mais elevada do PBZ induziu uma maior redução nas trocas gasosas da mangueira. Segundo Taiz e Zeiger (2013), a redução destas características reduz significativamente a produção da cultura, conforme observado neste trabalho. A diferença entre as características fisiológicas para as menores doses pode estar associado à época de avaliação da planta, frutificação, quando o efeito do PBZ poderia estar menor.

A viabilidade da aplicação do PBZ via sistema de irrigação encontrada neste trabalho corrobora com a citação de Coelho et al. (2001), que avaliando a distribuição do sistema radicular da mangueira sob irrigação localizada, verificaram elevada atividade do sistema radicular dento do bulbo molhado, o que reforça a recomendação de aplicação via sistema de irrigação como alternativa mais adequada de aplicação de fertilizantes, herbicidas e até dos reguladores vegetais ao solo. 

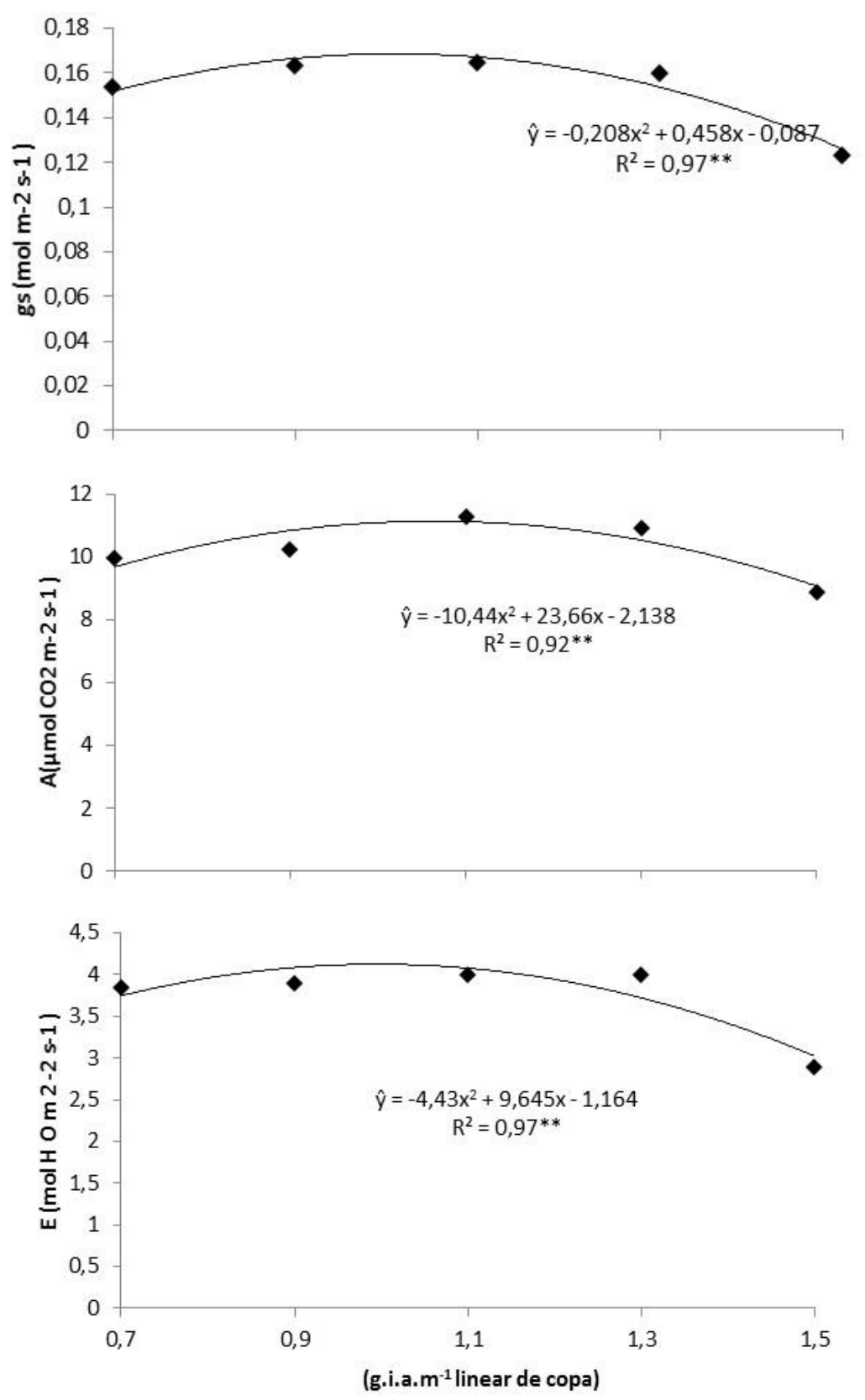

Figura 2. Condutância estomática $\left(\mathrm{g}_{\mathrm{s}}\right)$, transpiração $(\mathrm{A})$ e taxa de fotossíntese (E) da mangueira cultivar Keitt, em função das doses de paclobutrazol aplicadas via sistema de irrigação, na região do Submédio do São Francisco. Petrolina/PE. ** regressões significativas a $1 \%$ de probabilidade.

\section{CONCLUSÕES}

A aplicação do paclobutrazol via sistema de irrigação mostrou eficiência para o cultivo da mangueira 'Keitt', na região do Submédio São Francisco;

A dose de $0,7 \mathrm{~g}$ i.a. $\mathrm{PBZ} \mathrm{m}^{-1}$ (46\% da utilizada na aplicação convencional) via sistema de irrigação proporcionou um incremento de $32 \%$ na produtividade da mangueira 'Keitt'. 


\section{REFERÊNCIAS BIBLIOGRÁFICAS}

ALBUQUERQUE, J. A. S.; MEDINA, V. D.; MOUCO, M. A. do C. Indução floral. In: GENU, P. J. C.; PINTO, C. A. Q. (Ed.). A cultura da mangueira. Brasília, DF: Embrapa Informação Tecnológica, 2002. cap. 13, p. 259-276.

CARdoso, M. G. S.; SÃO JOSÉ, A. R.; VIANA, A. E. S.; MATSUMOTO, S. N.; REBOUÇAS, T. N. H.; Florescimento e frutificação de mangueira (Mangifera indica L.) cv. rosa promovidos por diferentes doses de paclobutrazol. Revista Brasileira de Fruticultura, Jaboticabal, v. 29, n. 2, p. 209-212, 2007.

CHATZIVAGIANNIS, M. A.; SÃO JOSÉ, A. B.; BOMFIM, M. P.; OLIVEIRA JÚNIOR, M. X. de;REBOUÇAS, T. N. H. Florescimento e produtividade de mangueira 'Boubon', 'Palmer'e 'Rosa'com uso de Paclobutrazol. Revista Iberoamericana de Tecnología Postcosecha, Hermosillo, v. 15, n. 1, p. 41-47, 2014.

COELHO, E. F.; OLIVEIRA, F. das C.; ARAÚJO E. C. E.; VASCONCELOS L. F. L.; LIMA D. M.; Distribuição do sistema radicular da mangueira sob irrigação localizada em solo arenoso de tabuleiros costeiros. Revista Brasileira Fruticultura, Jaboticabal, v. 23, n. 2, p. 250-256, 2001.

FERRARI, D. F.; SERGENT, E. A. Promoción de lafloración y frutificaciónen mango (Mangifera indica, L.) cv. Haden, com paclobutrazol. Revista de La Facultad de Agronomia, Maracay, v. 22, p. 9-17, 1996.

MOUCO, M. A. do C.; ONO, E. O. O.; RODRIGUES, J. D. Mango flower induction in the Brazilian Northeast Semi-arid with gibberellin synthesis inhibitors. Acta Horticulturae, Leuven, n. 884, p. 591-596, 2010.

RAMÍREZ, F.; DAVENPORT, T. L. Mango (Mangifera indica L.) flowering physiology.

Scientia Horticulturae, Amsterdam, v. 126, p. 65-72, 2010.

REIS, S.; R. J. B. Efeito de lâminas de irrigação e doses de PBZ na pós-colheita da mangueira cv. Haden no norte de Minas Gerais. Revista Brasileira de Agricultura Irrigada, Fortaleza, v. 5, n. 3, p. 214-224, 2011.

TAIZ, L.; ZEIGER, E. Fisiologia vegetal. 4.ed. Porto Alegre: Artmed, 2013. 820p 